\title{
ON THE HOMOGENEITY OF INFINITE PRODUCTS OF MANIFOLDS
}

\author{
T. GANEA ${ }^{1}$
}

Let $M$ be the Cartesian product of a countably infinite family of compact manifolds $M_{i}$. If every $M_{i}$ has a boundary, then $M$ is homogeneous [2]. Since a manifold without boundary is homogeneous, $M$ is also homogeneous if none of the $M_{i}$ has a boundary. It follows that $M$ is homogeneous if none or if infinitely many of the $M_{i}$ have a boundary. Answering a question raised by R. D. Anderson concerning the remaining case, we prove

THEOREM. The Cartesian product of a countably infinite family of compact manifolds of which finitely many have a boundary is not homogeneous.

Since a finite product of manifolds with boundary is, in turn, a manifold with boundary, we may assume that the product contains precisely one manifold with boundary. The fact that the product is countable is irrelevant: our proof applies to any infinite (or finite) product. We assume our manifolds to be Hausdorff spaces. A space is homogeneous if for any pair of points there is a homeomorphism of the space onto itself carrying one of the points into the other. A space $X$ is essential [1, p. 519] if it cannot be deformed into a proper subset, i.e., if any homotopy $h_{t}: X \rightarrow X$ with $h_{0}=1$ necessarily satisfies $h_{1}(X)$ $=X$; here and throughout the paper, 1 stands for the identity map.

Lемма 1. Let $X$ be the Cartesian product of a countable family of compact Hausdorff spaces $X_{i}$. If every finite product of spaces $X_{i}$ is essential, then $X$ itself is essential.

Proof. Suppose the contrary and let $h_{t}: X \rightarrow X$ satisfy $h_{0}=1$, $h_{1}(X)=C \neq X$. Then $U=X-C$ is a nonvoid open subset of $X$ and, as such, contains a subset of the form $a_{1} \times \cdots \times a_{n} \times X_{n+1}$ $\times X_{n+2} \times \cdots$ for a sufficiently large $n \geqq 1$ and suitable points $a_{i} \in X_{i}, 1 \leqq i \leqq n$. Select points $b_{i} \in X_{i}$ for $i \geqq n+1$, and consider the composite

$$
H_{\imath}: Y \stackrel{j}{\rightarrow} X \stackrel{h_{\iota}}{\rightarrow} X \stackrel{p}{\rightarrow} Y
$$

where $Y=\prod_{1 \leqslant i \leqslant n} X_{i}, j$ embeds $Y$ as the subset $Y \times b_{n+1} \times b_{n+2} \times \cdots$

Received by the editors February 18, 1966.

1 This work was partially supported by NSF GP-3902. 
of $X$, and $p$ projects onto the first $n$ factors. Then, $H_{0}=1$ and $H_{1}(Y) \subset p(C)$. But $a_{1} \times \cdots \times a_{n} \notin p(C)$; for, the contrary yields a point $c=a_{1} \times \cdots \times a_{n} \times c_{n+1} \times c_{n+2} \times \cdots \in C$ so that $c \in U$, and this is impossible since $C \cap U=\varnothing$. Thus, $Y$ fails to be essential and the result is proved.

A point $x \in X$ is unstable $[1$, p. 523] if for any open neighborhood $U$ of $x$ in $X$ there is a homotopy $h_{t}: X \rightarrow X$ with

$$
h_{0}=1, \quad h_{t} \mid X-U=1, \quad h_{t}(U) \subset U, \quad h_{1}(X) \neq X .
$$

A point which is not unstable is called stable $[1$, p. 523]. We denote by $\bar{U}$ the closure of any subset $U$. Also, $\left(B^{n}, E^{n}\right)$ stands for the pair consisting of the closed unit ball and its interior in Euclidean $n$-space.

Lemma 2. Let $M$ be the Cartesian product of a countable family of compact manifolds without boundary. Let $X$ be a compact Hausdorff space and suppose $x \in X$ has an open neighborhood $U$ such that the pair $(\bar{U}, U)$ is homeomorphic to $\left(B^{n}, E^{n}\right)$. Then, the point $x \times m$ is stable in $X \times M$ for any $m \in M$.

Proof. Let $S$ be the quotient space obtained from $X$ by shrinking to a point the subset $X-U$; let $f: X \rightarrow S$ be the identification map. Suppose $x \times m$ is unstable and let $h_{t}: X \times M \rightarrow X \times M$ be a homotopy satisfying the conditions (1) with respect to the neighborhood $U \times M$ of $x \times m$. It is readily seen that there exists a unique homotopy $H_{t}$ yielding commutativity in the diagram

$$
\begin{gathered}
S \times M \stackrel{H_{t}}{\longrightarrow} S \times M \\
\uparrow f \times 1 h_{t} \quad \uparrow f \times 1 \\
X \times M \stackrel{\longrightarrow}{\longrightarrow} \times M
\end{gathered}
$$

Obviously, $H_{0}=1$. There is a point $a \times b \notin h_{1}(X \times M)$, and it necessarily lies in $U \times M$. Hence, $f(a) \times b \notin H_{1}(S \times M)$ and $S \times M$ is proved to be inessential. However, $S$ is homeomorphic to an $n$-sphere, any finite product of compact manifolds without boundary is a compact manifold without boundary, and any such $q$-dimensional manifold $Q$ is essential since its Cech cohomology group $H^{q}\left(Q, Z_{2}\right) \neq 0$ whereas $H^{q}\left(P, Z_{2}\right)=0$ for any closed proper subset $P \subset Q$. Thus, by Lemma 1 , $S \times M$ is essential and Lemma 2 is proved.

LemMA 3. Let $X$ be any space and suppose $Y$ is completely regular. If $a \in X$ is unstable, then $a \times b$ is unstable in $X \times Y$ for any $b \in Y$.

Proof. Let $W$ be any neighborhood of $a \times b$ in $X \times Y$; we may assume that $W=U \times V$ where $U$ and $V$ are open in $X$ and $Y$. Let 
$h_{t}: X \rightarrow X$ be a homotopy satisfying (1) with respect to $U$. Select a continuous function $r: Y \rightarrow I$ with $r(b)=1, r(Y-V)=0$. Then, $a \times b$ is readily seen to be unstable using the homotopy $H_{t}: X \times Y \rightarrow X \times Y$ given by $H_{t}(x \times y)=h_{t r(y)}(x) \times y$.

To prove the theorem, it only remains to note that an inner point of a manifold with boundary satisfies the assumption in Lemma 2, whereas a boundary point is obviously unstable. Thus, the Cartesian product discussed has both stable and unstable points, and cannot be homogeneous.

\section{REFERENCES}

1. P. Alexandroff and H. Hopf, Topologie, Springer, Berlin, 1935.

2. M. K. Fort, Jr., Homogeneity of infinite products of manifolds with boundary, Pacific J. Math. 12 (1962), 879-884.

UNIVERSITY OF WASHINGTON 